Swarthmore College

Works

$10-21-2013$

\title{
Aggregation, Pretransitional Behavior, And Optical Properties In The Isotropic Phase Of Lyotropic Chromonic Liquid Crystals Studied In High Magnetic Fields
}

T. Ostapenko

Y. A. Nastishin

Peter J. Collings

Swarthmore College, pcollin1@swarthmore.edu

S. N. Sprunt

O D. Lavrentovich

See next page for additional authors

Follow this and additional works at: https://works.swarthmore.edu/fac-physics

Part of the Physics Commons

Let us know how access to these works benefits you

\section{Recommended Citation}

T. Ostapenko, Y. A. Nastishin, Peter J. Collings, S. N. Sprunt, O D. Lavrentovich, and J. T. Gleeson. (2013). "Aggregation, Pretransitional Behavior, And Optical Properties In The Isotropic Phase Of Lyotropic Chromonic Liquid Crystals Studied In High Magnetic Fields". Soft Matter. Volume 9, Issue 39. 9487-9498. DOI: $10.1039 / \mathrm{c} 3 \mathrm{sm} 51819 \mathrm{a}$

https://works.swarthmore.edu/fac-physics/170

This work is brought to you for free by Swarthmore College Libraries' Works. It has been accepted for inclusion in Physics \& Astronomy Faculty Works by an authorized administrator of Works. For more information, please contact myworks@swarthmore.edu. 


\section{Authors}

T. Ostapenko, Y. A. Nastishin, Peter J. Collings, S. N. Sprunt, O D. Lavrentovich, and J. T. Gleeson 
Cite this: Soft Matter, 2013, 9, 9487

Received 3rd July 2013

Accepted 20th August 2013

DOI: $10.1039 / \mathrm{c} 3 \mathrm{sm} 51819 a$

www.rsc.org/softmatter

\section{Aggregation, pretransitional behavior, and optical properties in the isotropic phase of lyotropic chromonic liquid crystals studied in high magnetic fields}

\author{
Tanya Ostapenko, $\dagger^{\star a}$ Yuriy A. Nastishin, ${ }^{\text {bc }}$ Peter J. Collings, ${ }^{d}$ Samuel N. Sprunt, ${ }^{a}$ \\ Oleg D. Lavrentovich be and J. T. Gleeson ${ }^{a}$
}

\begin{abstract}
We report results on the high-field magneto-optical response of four aqueous, lyotropic, chromonic liquid crystal formulations in the isotropic phase. Measurements of the field-induced birefringence at temperatures above the isotropic-nematic coexistence region at high magnetic fields reveal qualitative differences in different materials; these differences can be attributed to the nature of aggregation and are discussed within the context of competing aggregation models. Extending these measurements to very high fields and large optical phase differences reveals the presence of an unexpected optical phenomenon: magnetic field-induced circular birefringence, measured in the Voigt geometry, in a system containing no molecularly chiral species. Possible origins of this effect are discussed.
\end{abstract}

\section{Introduction}

Lyotropic chromonic liquid crystals (LCLCs) are a class of complex fluids that holds potential for understanding both molecular interactions and supramolecular assembly. Their basic structure consists of relatively rigid aromatic cores, with hydrophilic ionic groups positioned around the outside of the molecule. ${ }^{1,2}$ In comparison, amphiphilic mesogens of typical surfactant type are rod-like, with a hydrophilic group positioned at one end of the relatively flexible hydrophobic aliphatic chain. Both chromonic and amphiphilic molecules, when dispersed in water, self-assemble into aggregates, but differences in their molecular structure contribute to differences in the molecular organization of the aggregates, the character of the aggregation, and aggregate ordering.

One essential difference between amphiphilic and chromonic aggregates is their molecular packing. Amphiphilic molecules in micelles can diffuse readily along the surface, essentially forming a two-dimensional liquid. In contrast, the molecular packing inside a chromonic

\footnotetext{
${ }^{a}$ Department of Physics, Kent State University, Kent, OH 44242, USA. E-mail: tostapen@kent.edu; ssprunt@kent.edu; jgleeson@kent.edu; Fax: +1 330672 2959; Tel: +1 3306722246

${ }^{b}$ Liquid Crystal Institute, Kent State University, Kent, OH 44242, USA

'Institute of Physical Optics, 23 Dragomanov str., Lviv, 79005, Ukraine

${ }^{d}$ Department of Physics \& Astronomy, Swarthmore College, Swarthmore, PA 19081, USA

${ }^{e}$ Chemical Physics Interdisciplinary Program, Kent State University, Kent, OH 44242, USA

$\uparrow$ Current address: Institute of Experimental Physics, Otto-von-GuerickeUniversität, 39106 Magdeburg, Germany; E-mail: tanya.ostapenko@ovgu.de
}

aggregate much more closely resembles a structure where molecules possess some positional order along a single direction. Furthermore, self-assembly is substantially different between chromonic aggregates and micelles; in most cases, the plank-like chromonics stack face-to-face into rod-like aggregates with open ends, such that the free energy per molecule is independent of the aggregate size. This implies that there is no critical micelle concentration (CMC) or temperature (СMT) for aggregation; this type of self-assembly is commonly referred to as isodesmic. Though there is a prediction ${ }^{3}$ that dimers might form at a critical concentration (which we will also label CMC), most chromonics evidently assemble isodesmically.

One LCLC that has been extensively studied is disodium cromoglycate (DSCG), which was originally developed as an antiasthmatic drug. ${ }^{4}$ Other examples of LCLCs include dyes such as Sunset Yellow (SSY), Violet 20, Direct Blue 67 and Blue $27 .{ }^{5}$ Despite numerous studies done on the LCLC aggregation process through various experimental techniques (see ref. 6-8 for recent reviews), there are still unanswered questions as to how the aggregation mechanism proceeds. LCLC molecular self-assembly depends on factors such as molecular structure, concentration, temperature, solvent polarity, and ionic strength. ${ }^{2,9}$ It is thought that the cause of face-to-face aggregation is the $\pi-\pi$ interaction of aromatic cores, but other causes can include any noncovalent interactions that the molecules may have. ${ }^{9}$ Some of these studies have explored the molecular behavior in the nematic phase, but there are only a few studies $^{5,10}$ of the pretransitional behavior as the coexistence region of liquid crystal and the isotropic liquid is approached from the pure isotropic phase. 
Results from X-ray scattering and optical techniques suggested that the nematic liquid crystal phase of SSY shares many of the same properties as DSCG, including isodesmic aggregation and columnar aggregates. ${ }^{5}$ Determining the aggregate nature in the isotropic phase is more difficult. In this case, it is possible to induce optical anisotropy by application of an external field, such as a high magnetic field, and record it with high resolution in order to probe the shape anisotropy of the aggregates. Since the molecular planes in the aggregate are perpendicular, or nearly perpendicular, to the stacking direction, and since the molecular diamagnetic anisotropy is typically negative, the molecular planes tend to align parallel to an applied magnetic field, so that the long axes of the aggregates orient perpendicular to the field direction.

Such field-induced alignment leads to induced birefringence, i.e. the Cotton-Mouton (CM) effect. ${ }^{11}$ However, results from this technique have produced conflicting reports concerning the nature of aggregation, especially in DSCG: Champion and Meeten $^{\mathbf{1 2}}$ studied the magnetic field-induced birefringence of DSCG and saw behavior that was consistent with isodesmic aggregation. On the other hand, Luoma ${ }^{13}$ investigated the magnetic field-induced birefringence of both DSCG and Sunset Yellow, finding isodesmic behavior only in the latter. Moreover, non-isodesmic behavior for the DSCG solution was claimed from the results of light scattering ${ }^{14}$ and absorption studies. ${ }^{6}$ Thus, the precise nature of aggregation, especially in DSCG, remains an open question.

To investigate experimentally the nature of the aggregates and aggregation process in the isotropic phase of LCLCs, one must account for the effects of pretransitional nematic ordering. In standard thermotropic liquid crystals, the pretransitional short-range order in the isotropic phase is described well by Landau-deGennes theory. ${ }^{15}$ According to this theory, the Cotton-Mouton coefficient decreases with temperature as $1 /\left(T-T^{*}\right)$, where $T^{*}$ is the thermodynamic stability (supercooling) limit of the isotropic phase. Since the mean LCLC aggregate length, and therefore aspect ratio, also change with temperature, one may anticipate that $T^{*}$ itself is a temperature-dependent parameter. If the aggregation process is isodesmic, the mean aggregate length decreases continuously with increasing temperature, approaching molecular dimensions only at a very high temperature. On the other hand, in a non-isodesmic process, aggregates could form, or lengthen, abruptly at some critical temperature in the isotropic phase. In either case, the Cotton-Mouton coefficient should have a temperature dependence reflecting both pretransitional behavior $\left[1 /\left(T-T^{*}(T)\right)\right]$ and a change in the form (shape) birefringence due to the temperature-dependent length.

In the work reported here, we used high-field magnetic birefringence to probe aggregation and pretransitional nematic ordering in four different LCLC systems above the biphasic (nematic-isotropic coexistence) region. In addition to testing the Landau-deGennes prediction in LCLC systems, our results provide insight into the character (isodesmic versus non-isodesmic) of the temperature-dependent aggregate growth.

\section{Experimental details}

Four different LCLC systems were studied for this work (Fig. 1). These four compounds were selected for two reasons: (1) they are among the most studied of the chromonic systems, and (2) based on these studies, they represent both a range of assembly structures and differing behavior. All were prepared as aqueous solutions: (i) $12 \mathrm{wt} \%$ and $14 \mathrm{wt} \%$ 5-[3-(2-carboxy-4-oxochromen6-yl)oxy-2-hydroxypropoxy]-4-oxochromene-2-carboxylic acid (DSCG), from Spectrum, Inc. (original purity = 98\%), (ii) $25 \mathrm{wt} \%$ of the disodium salt of 6-hydroxy-5-[(4-sulfophenyl)azo]-2naphthalenesulfonic acid (Sunset Yellow or SSY) from Sigma Aldrich (original purity $=95.7 \%$ ) (iii) $2.5 \mathrm{wt} \%$ Bordeaux dye (BD), the product of the sulfonation of the cis-dibenzimidolazole derivative of naphthalenetetracarboxylic acid, from Optiva, Inc., and (iv) 3.65 wt\% 6,15-disulfonicacid-7,16-dichloro-6,15dihydrodinaptho[2,3-a; 2,3-h]phenazine-5,9,14,18-tetraone diammonium salt known as Blue 27 (B27), from Optiva, Inc.

The LCLC concentrations were chosen to yield clearing point temperatures $T_{N+I}$ (upper limit of the coexistence region) near room temperature. Since $T_{N+I}$ for Sunset Yellow depends very sensitively on concentration, we opted for a particularly low clearing point to allow heating well above this temperature without water loss. As a result, we were able to study temperatures 30 degrees or more above the transition without water evaporation being a problem; this was verified by checking that

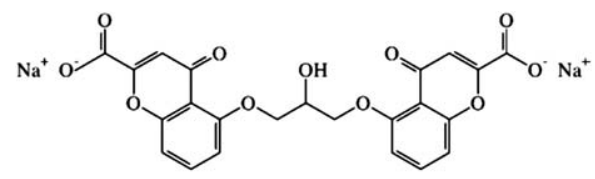

(a)

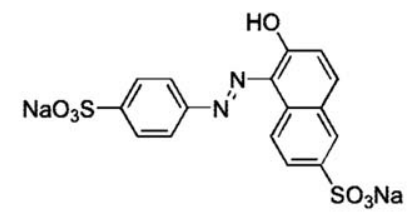

(b)

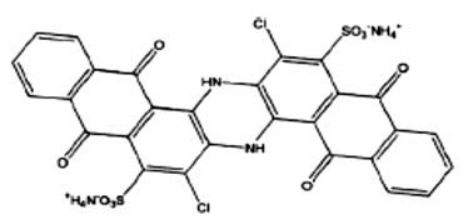

(c)

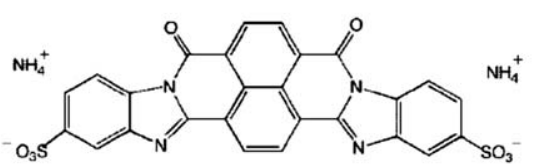

(d)

Fig. 1 LCLC materials used for aggregation studies. (a) Disodium cromoglycate (DSCG), ${ }^{16}$ (b) Sunset Yellow (SSY), ${ }^{17}$ (c) Blue $27,{ }^{18}$ (d) Bordeaux dye. ${ }^{19}$ 
there was no change in the clearing temperature. For high-field measurements, DSCG, Bordeaux and Sunset Yellow were sealed in $1 \mathrm{~mm}$ path length, B270 crown glass cuvettes. Blue 27, because of its greater absorbance, was also sealed in a $1 \mathrm{~mm}$ cuvette, but the thickness was decreased by adding borosilicate slides to reduce the optical path length to $0.46 \mathrm{~mm}$.

We determined $T_{N+I}$ by cooling the sample from high temperatures and monitoring the intensities of both backscattered and transmitted light in zero magnetic field. In the coexistence region, the scattering is much larger than the transmission, due to the presence of nematic droplets, from which the light scatters strongly. We took the transition temperature to be the temperature at which the transmission went to zero and scattering reached a plateau. The transition temperatures, using our setup, were: $T_{N+I}^{\mathrm{B} 27} \approx 25^{\circ} \mathrm{C}, T_{N+I}^{\mathrm{BD}} \approx 26.5^{\circ}$ $\mathrm{C}, T_{N+I}^{\mathrm{DSCG}}(12 \mathrm{wt} \%) \approx 26^{\circ} \mathrm{C}, T_{N+I}^{\mathrm{DSCG}}(14 \mathrm{wt} \%) \approx 30.5^{\circ} \mathrm{C}$, and $T_{N+I}^{\mathrm{SSY}} \approx 15^{\circ} \mathrm{C}$.

High resolution measurements of field-induced optical phase shifts were conducted in the isotropic phase of the LCLC samples using the classic technique combining photoelastic phase modulation and lock-in signal detection, as described in ref. 20. Data analysis was performed with updated calibration characteristics for the photoelastic modulator; details of the calibration procedure can be found in ref. 21. Fig. 2 illustrates the experimental setup. The sample was inserted into a twostage, temperature-controlled brass oven with $\pm 10 \mathrm{mK}$ stability. The oven was suspended at the midpoint in the bore of the 31 Tesla solenoid at the National High Magnetic Field Laboratory
(Cell 5). A diagram of the sample geometry appears in Fig. 3; in this arrangement, the magnetic field is perpendicular to the optical path (further details are provided in ref. 22). Any stray birefringence induced by the sample glass, steering mirrors, or other optics was compensated out using a Soleil-Babinet compensator. The light source used to study Sunset Yellow, Bordeaux dye, ${ }^{19}$ and DSCG was a $632.8 \mathrm{~nm}$ helium-neon laser; there is negligible absorption by these solutions at this wavelength. For Blue 27, we used an Argon-ion laser set in multi-line mode and a monochromator to select the line at a wavelength of $457.9 \mathrm{~nm}$. Absorption at this wavelength was significant, but accurate measurements of optical phase shifts were still feasible.

Two experimental protocols were followed: (1) field sweeps in which the field was ramped from 0 to 30 Tesla at a rate of 10.125 Tesla $\min ^{-1}$ and at fixed temperatures starting 1-2 degrees above the upper limit of the coexistence region, and (2) temperature sweeps in which the temperature was ramped above $T_{N+I}$ while holding the field fixed between 25 and 30 Tesla. Throughout both protocols, the intensity of transmitted light was simultaneously monitored at three frequencies: DC, $1 f$ and $2 f$, where $f=50 \mathrm{kHz}$ is the photoelastic modulation frequency. The ratio of the $1 f$ to $2 f$ amplitude is proportional to $\tan \varphi$, where $\varphi$ is the phase difference between the ordinary and extraordinary rays traversing the sample. The phase difference is related to the induced birefringence (refractive index anisotropy $\Delta n)$ according to $\varphi=(2 \pi d \Delta n) / \lambda$, where $d$ is the sample thickness and $\lambda$ is the vacuum wavelength.

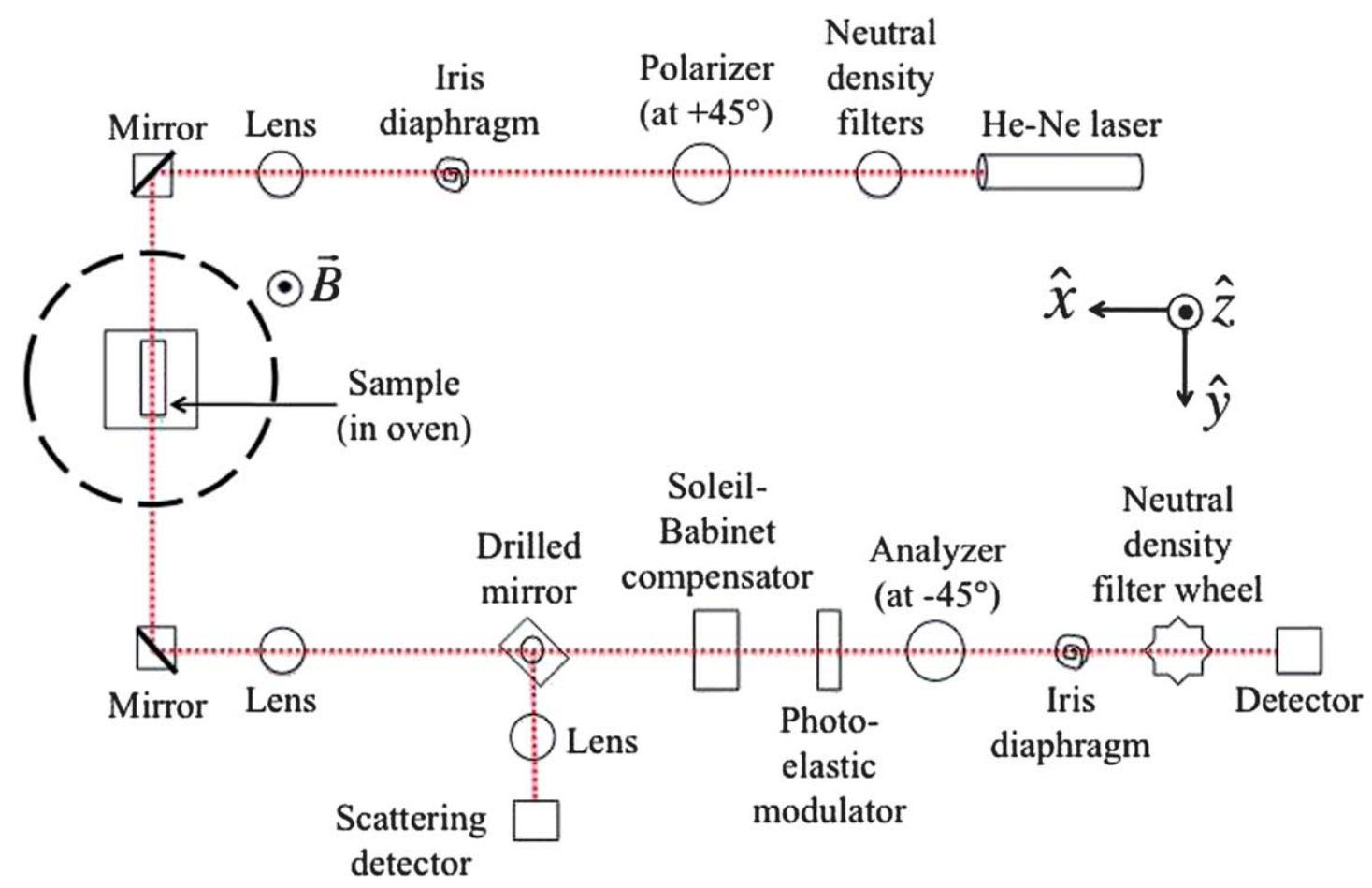

Fig. 2 (Color online) Optical setup for pathlength perpendicular to the magnetic field; the coordinate system is indicated to the right. The dotted circle denotes the magnet, with the magnetic field pointing in the $\hat{z}$-direction (out of the page). The two mirrors attached to the oven that were used to direct the laser beam through the sample are not explicitly shown. ${ }^{21}$ 


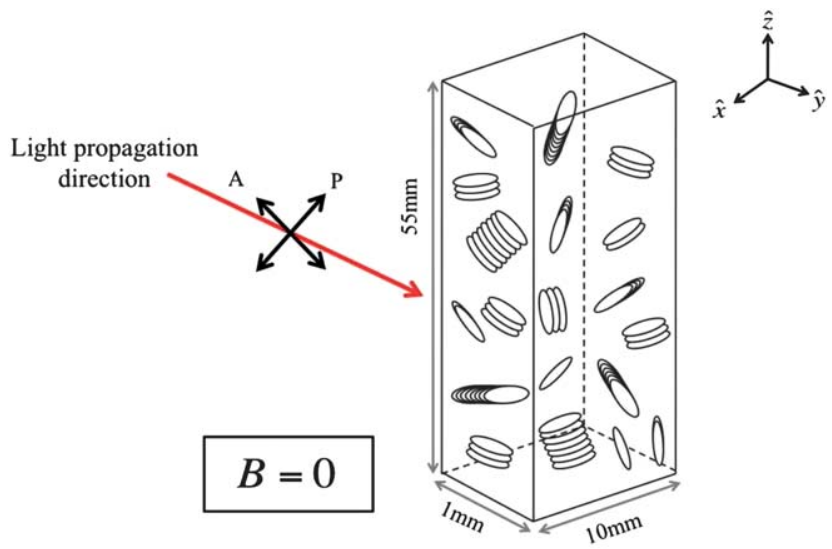

(a)

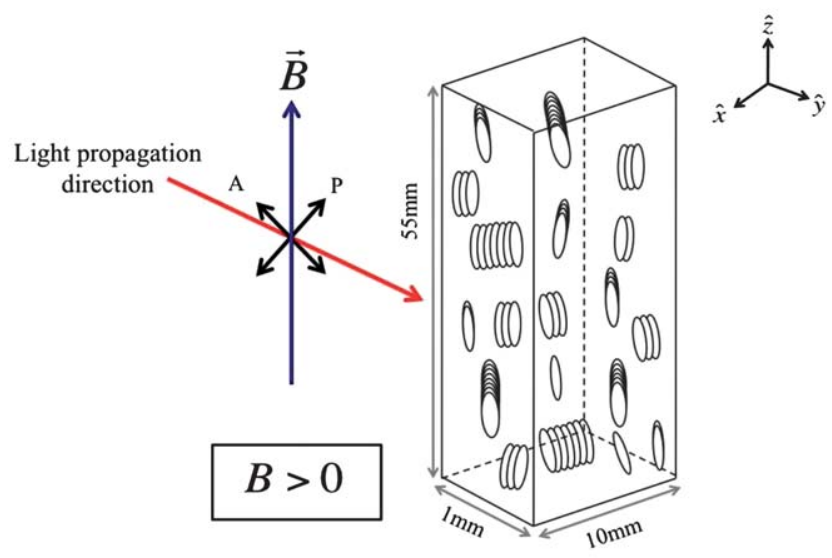

(b)

Fig. 3 (Color online) Sample geometry, with light passing through the sample linearly polarized at $45^{\circ}$. (a) When $B=0$, the molecules/aggregates may align in any direction at temperatures above $T_{N+1}$. As the temperature approaches this transition, the number of aggregates increases. (b) When $B>0$, the molecules align parallel to the applied magnetic field, causing the aggregates' long axis to align perpendicular to the magnetic field; i.e., the material has a negative diamagnetic anisotropy. Due to this induced anisotropy, a phase difference is present in the material, increasing as the field increases and the molecules become more aligned. This effect is more apparent when the temperature is closer to $T_{N+1}$.

\section{Results and discussion}

\subsection{Low field behavior and temperature dependence of the Cotton-Mouton coefficient}

The Cotton-Mouton effect in a diamagnetic medium is a quadratic relationship between $\Delta n$ and the applied magnetic field $B$ given by: ${ }^{11}$

$$
\Delta n=C \lambda B^{2} .
$$

Here, $C=C(T)$ is the temperature-dependent Cotton-Mouton coefficient, and $\lambda$ is the wavelength of the laser used. This relation applies for values of $B$ for which $\Delta n$ is well below the saturated value. Since the magnetic permeability of the LCLC differs negligibly from the vacuum permeability, $B$ can be taken to be the measured field in the empty bore.
In Fig. 4, we plot $\Delta n$ as a function of $B^{2}$ for different temperatures in the isotropic phase (above $T_{N+I}$ ) for the four LCLC systems studied. All four show $\Delta n \propto B^{2}$ at sufficiently small $B$ or sufficiently high temperature relative to $T_{N+I}$. The values of $\Delta n$ for Blue 27 are 30-50 times lower than the other LCLC materials. The behavior is similar to the difference in birefringence seen in the homogeneous nematic phases of these compounds. ${ }^{18}$ We attribute this to the fact that the best choice of optical wavelength available to us still lies in the wings

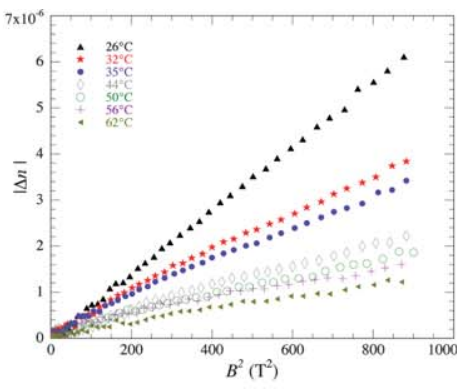

(a)

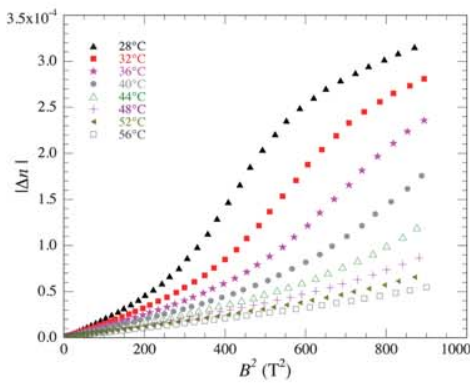

(b)

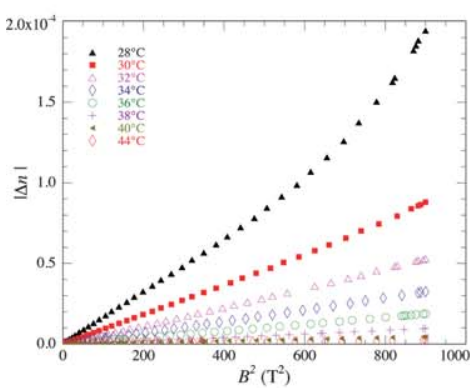

(c)

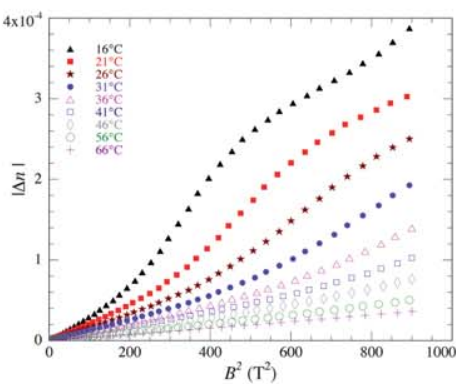

(d)

Fig. 4 (Color online) $|\Delta n|$ versus $B^{2}$ for (a) Blue 27, (b) Bordeaux dye, (c) DSCG, and (d) Sunset Yellow. Only selected temperatures for each material are shown for clarity. 
of resonant absorption of Blue 27, and thus the real part of the complex refractive index may be anomalously low. ${ }^{18}$ By fitting the low field data in Fig. 4 to the linear relationship presented in eqn (1), we extracted the Cotton-Mouton coefficient $C=\left(\frac{\mathrm{d} \Delta n}{\mathrm{~d} B^{2}}\right)_{B \rightarrow 0}$. At higher values of $B(B \geq 10$ Tesla $)$ and for $T$ close to $T_{N+I}$, we note that $\Delta n$ is not linear in $B^{2}$ for Bordeaux dye and Sunset Yellow. Such a nonlinear dependence at high field was previously observed in colloidal suspensions, ${ }^{23}$ goethite $\alpha$ FeOOH nanorods,${ }^{24}$ and hard colloidal platelets. ${ }^{25}$ The deviation from $\Delta n \propto B^{2}$ in those cases is caused by phase separation, a field-induced transition, or competition between magnetic properties. In Section 3.2 below, we will address the question of what mechanism in LCLCs might cause the departure from the classical Cotton-Mouton effect at high fields.

Fig. 5 displays the dependence of $C$ on $T$ in the isotropic phase of the four LCLCs studied. These data are revealing of the nature of the isotropic-nematic transition and potentially of the aggregation mechanism. For Blue 27, Bordeaux dye, and Sunset Yellow, $C(T)$ tends gradually away from zero as the temperature is decreased. However, in the case of DSCG, $C(T)$ rapidly increases from a flat value consistent with zero, within the precision of our measurement.

The prediction from Landau-deGennes theory for the pretransitional temperature dependence of $C(T)$ above the isotropic to nematic transition is:

$$
C(T)=\frac{C_{\max }}{T-T^{*}},
$$

where $C_{\max }=\frac{2}{9} \frac{\Delta n_{\max } \Delta \chi_{\max }}{a_{0}}, \Delta n_{\max }$ and $\Delta \chi_{\max }$ are the birefringence and anisotropy of magnetic susceptibility for the fully ordered uniaxial nematic state, respectively, and $a_{0}$ is the coefficient of the lowest order (quadratic) term in the Landaude Gennes expansion of the isotropic phase free energy in terms of the nematic order parameter. In thermotropic nematics,

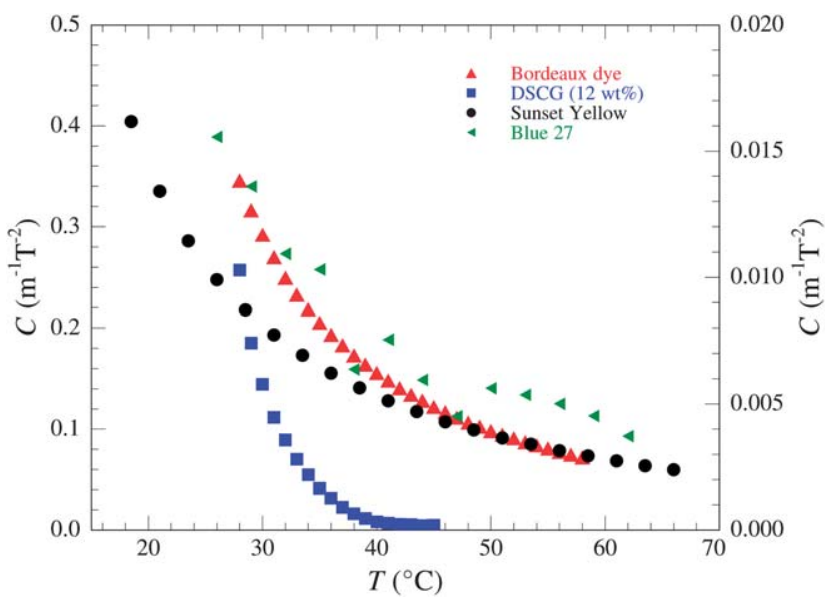

Fig. 5 (Color online) The classical Cotton-Mouton coefficient versus T for Blue 27 (sideways triangles), Bordeaux dye (triangles), DSCG (squares), and Sunset Yellow (circles). Since the data for Blue 27 are substantially lower than that for the other three systems, it is plotted against the scale on the right axis.

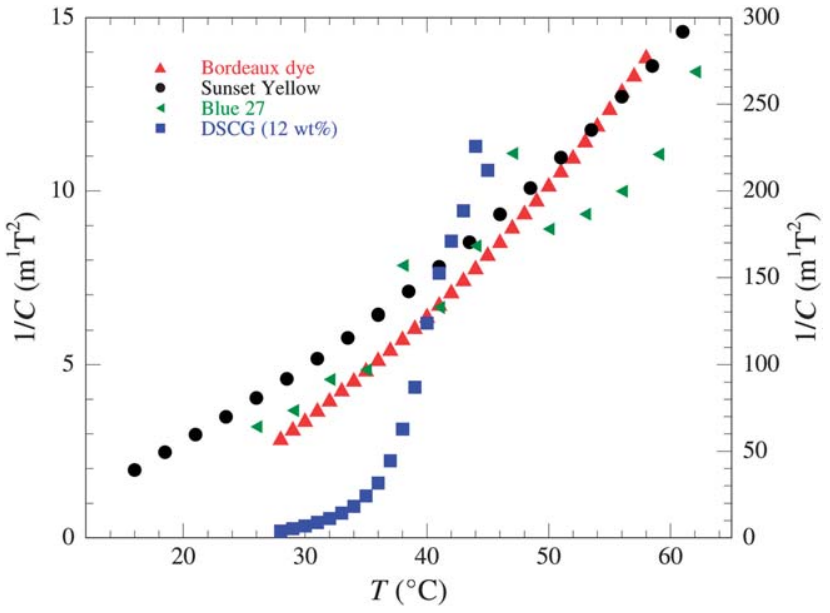

Fig. 6 (Color online) 1/C versus $T$ for all four systems. The data for Blue 27 and DSCG are plotted against the right axis. For DSCG, $1 / C$ is non-linear, but for the other three materials, we note that there is a nearly linear trend.

the supercooling limit $T^{*}$ of the isotropic phase is a constant (i.e. a material property). However, our data are not fully described by the simple prediction in eqn (2) (Fig. 6).

To develop an expression for $C(T)$ applicable to LCLCs, we must account for the temperature-dependent length of the aggregates - and specifically its potential effect on the form birefringence, the birefringence resulting from anisotropy on scales larger than the molecular scale,${ }^{26-28}$ of the LCLC solution - in addition to the impact of pretransitional nematic order. Since the saturated refractive index anisotropy in the nematic phase of LCLCs is small compared to thermotropics $\left(\Delta n_{\max } \simeq\right.$ 0.02 versus 0.15 ), the aspect ratio of the aggregates is much larger (up to $100: 1$ versus $6: 1$ ). Furthermore, since the index contrast between the aggregates and aqueous solvent is not small ( $n_{\text {LCLC }}-n_{\text {water }} \simeq 0.2$ ), form birefringence could be a significant contributor to the field-induced $\Delta n$ we measure, in addition to the influence of molecular anisotropy. The form birefringence of a uniaxial anisotropic particle is generally a complicated function of the particle aspect ratio, $L(T) / d$. We take $L$ as the temperature-dependent mean length and $d$ as the (fixed) cross-sectional diameter of the LCLC aggregates.

In order to keep the analysis simple, we will model the aggregates as spheroidal objects with $L / d \gg 1$ giving a long, thin prolate shape, approaching a cylinder in the limit $L / d \rightarrow \infty$, and $L / d<1$ giving an oblate shape. We will also assume that the aggregates are continuous media, with anisotropic dielectric constants $\varepsilon_{12}$ and $\varepsilon_{3}$ surrounded by a solvent medium with isotropic dielectric constant $\varepsilon_{\text {sol }}$. The 3 direction corresponds to the axis of rotational symmetry of an aggregate and 1,2 to the plane perpendicular to 3 . In the case of spheroids, the electric field is uniform inside, and the refractive index anisotropy $\Delta n_{\max }$ comprising both form and molecular contributions for a fully aligned LCLC may be written in a simple form for $\Delta_{\max } \ll 1$ as: $^{26}$

$$
\Delta n_{\max }=\frac{f}{2 \sqrt{\bar{\varepsilon}}}\left[\left(\frac{\varepsilon_{\|}-\varepsilon_{\mathrm{sol}}}{1+k_{\|} D_{\|}}\right)-\left(\frac{\varepsilon_{\perp}-\varepsilon_{\mathrm{sol}}}{1+k_{\perp} D_{\perp}}\right)\right]
$$

where $f$ is the volume fraction of the LCLC molecules, $\bar{\varepsilon}$ is the dielectric constant of an isotropic distribution of pure LCLC 
molecules, $k_{\|, \perp}=(1-f)\left(\frac{\varepsilon_{\|, \perp}}{\varepsilon_{\text {sol }}}-1\right)$, and $D_{\|}$and $D_{\perp}$ are depolarization factors. Here, the direction $\|$ corresponds to the applied field $B$. Since $\Delta n$ only reaches $\sim 0.02$ in the nematic phase of LCLCs, the condition $\Delta n_{\max } \ll 1$ is fulfilled.

Due to the negative diamagnetic anisotropy, the aggregate long axes all lie degenerately in the plane perpendicular to $B$ when the system is fully aligned by $B$. As the inter-aggregate spacing is much smaller than the optical wavelength, we write $D_{\|}=D_{12}$ and $D_{\perp}=\left(D_{12}+D_{3}\right) / 2$, where $D_{12}$ and $D_{3}$ are the depolarization factors for a spheroid. Similarly, we take $\varepsilon_{\|}=\varepsilon_{12}$ and $\varepsilon_{\perp}=\left(\varepsilon_{12}+\varepsilon_{3}\right) / 2$. The depolarization factors are given by; ${ }^{29}$ for $m \equiv L / d>1$ :

$$
\begin{aligned}
D_{12} & =\frac{m}{2\left(m^{2}-1\right)}\left[m-\frac{1}{2 \sqrt{m^{2}-1}} \ln \left(\frac{m+\sqrt{m^{2}-1}}{m-\sqrt{m^{2}-1}}\right)\right] \\
D_{3} & =\frac{1}{m^{2}-1}\left[\frac{m}{2 \sqrt{m^{2}-1}} \ln \left(\frac{m+\sqrt{m^{2}-1}}{m-\sqrt{m^{2}-1}}\right)-1\right]
\end{aligned}
$$

for $m=1$ :

$$
D_{12}=D_{3}=\frac{1}{3}
$$

and for $m<1$ :

$$
\begin{gathered}
D_{12}=\frac{m}{2\left(1-m^{2}\right)}\left[\frac{1}{\sqrt{1-m^{2}}} \sin ^{-1}\left(\sqrt{1-m^{2}}\right)-m\right] \\
D_{3}=\frac{1}{1-m^{2}}\left[1-\frac{m}{\sqrt{1-m^{2}}} \sin ^{-1}\left(\sqrt{1-m^{2}}\right)\right]
\end{gathered}
$$

For simplicity in what follows, we shall replace a statistical distribution in values of $m$ at each temperature with the mean value, $m \rightarrow \bar{m}=\bar{L}(T) / d$.

Although eqn (3) embodies a number of approximations, it captures the essential physical results. Namely, in the limit of no LCLC molecules $(f=0), \Delta n_{\max }$ vanishes, while in the case of pure LCLC ( $f=1$, no solvent), $\Delta n_{\max } \propto \varepsilon_{\|}-\varepsilon_{\perp}$. When there is no shape anisotropy $(m=1)$, and using the fact that $k_{\|},{ }_{\perp} \ll 1$ in this case, we again obtain $\Delta n_{\max } \propto \varepsilon_{\|}-\varepsilon_{\perp}$, i.e. the optical anisotropy is entirely due to anisotropic molecular polarizability.

Next, we must account for the pretransitional nematic order. We use the Landau-deGennes prediction (eqn (2)) together with a temperature-dependent $T^{*}$ arising due to the temperaturedependent $\bar{L} / d$. Following, ${ }^{\mathbf{1 0}}$ we write:

$$
T-T^{*}=T-T_{0}^{*}-\alpha_{2}\left(T-T_{0}^{*}\right)^{2}-\alpha_{3}\left(T-T_{0}^{*}\right)^{3},
$$

where $\alpha_{2}$ and $\alpha_{3}$ are constants, and $T_{0}^{*}$ corresponds to the supercooling limit of the isotropic phase for a uniform system of aggregates with mean length $\bar{L}\left(T_{0}^{*}\right)$. Combining eqn (2), (3) and (9) yields:

$$
\begin{aligned}
C(T)= & \frac{2}{18} \Delta \chi_{\max } f\left[\left(\frac{\varepsilon_{\|}-\varepsilon_{\text {sol }}}{1+k_{\|} D_{\|}}\right)-\left(\frac{\varepsilon_{\perp}-\varepsilon_{\text {sol }}}{1+k_{\perp} D_{\perp}}\right)\right] \\
& \times\left[T-T_{0}^{*}-\alpha_{2}\left(T-T_{0}^{*}\right)^{2}-\alpha_{3}\left(T-T_{0}^{*}\right)^{3}\right]^{-1}
\end{aligned}
$$

The final ingredient is an expression for $\bar{L}(T) / d$, which contributes in eqn (10) through the depolarization factors $D_{\|}$ and $D_{\perp}$. For an isodesmic aggregation process, one theoretically expects (ref. 30 and references therein) an Arrhenius-like temperature dependence:

$$
\frac{\bar{L}(T)}{d}=\frac{L_{0}}{d} e^{1 / 2} \phi^{1 / 2} \exp \left(\frac{E_{\mathrm{a}}}{2 k_{\mathrm{B}} T}\right)
$$

here, $E_{\mathrm{a}}$ is the "scission" energy, or the extra energy associated with the two end caps of an aggregate relative to the energy per molecule in the interior stack, $\phi$ is the volume fraction of chromonic molecules, $L_{0}$ is a length comparable to the intermolecular spacing $\left(L_{0} \sim 0.3 \mathrm{~nm}\right), e=\exp (1) \approx 2.718$, and $T$ is the absolute temperature. Typical values of the scission energy are $E_{\mathrm{a}} \sim 8--10 k_{\mathrm{B}} T .^{8}$ For variations around room temperature ( $\sim 300 \mathrm{~K})$, eqn $(11)$ gives a relatively weak, approximately linear temperature dependence. The analysis leading to eqn (11) will be reviewed below.

In Fig. 7, we display the results of fitting data for $C(T)$ for three of the materials studied, Blue 27, Bordeaux dye and Sunset Yellow, to eqn (10) in the form:

$$
\begin{aligned}
C(T)= & A \mid\left(\frac{1}{1+k_{\|} D_{\|}}\right)-\left(\frac{\Delta}{1+k_{\perp} D_{\perp}}\right) \\
& \times \mid\left[T-T_{0}^{*}-\alpha_{2}\left(T-T_{0}^{*}\right)^{2}-\alpha_{3}\left(T-T_{0}^{*}\right)^{3}\right]^{-1}
\end{aligned}
$$

Here, the absolute value is taken since our measurement of optical phase shift is not sensitive to sign, and eqn (11) describes the temperature dependence of $\bar{L} / d$ that enters in the depolarization factors. In the fits, we fixed $\Delta=\frac{\varepsilon_{\perp}-\varepsilon_{\text {sol }}}{\varepsilon_{\|}-\varepsilon_{\text {sol }}}$ to values between 0.9 and $1, k_{\|} \simeq k_{\perp}=0.2--0.25, E_{\mathrm{a}} / k_{\mathrm{B}}=3000 \mathrm{~K}$, and $L_{0} / d=0.3 .^{8}$ The parameters $A, T_{0}^{*}, \alpha_{2}, \alpha_{3}$ were set to reasonable starting values and then freely varied. As Fig. 7 shows, these fits accurately describe the data, and we conclude that these data are consistent with an isodesmic aggregation process in the isotropic phase of aqueous Sunset Yellow,

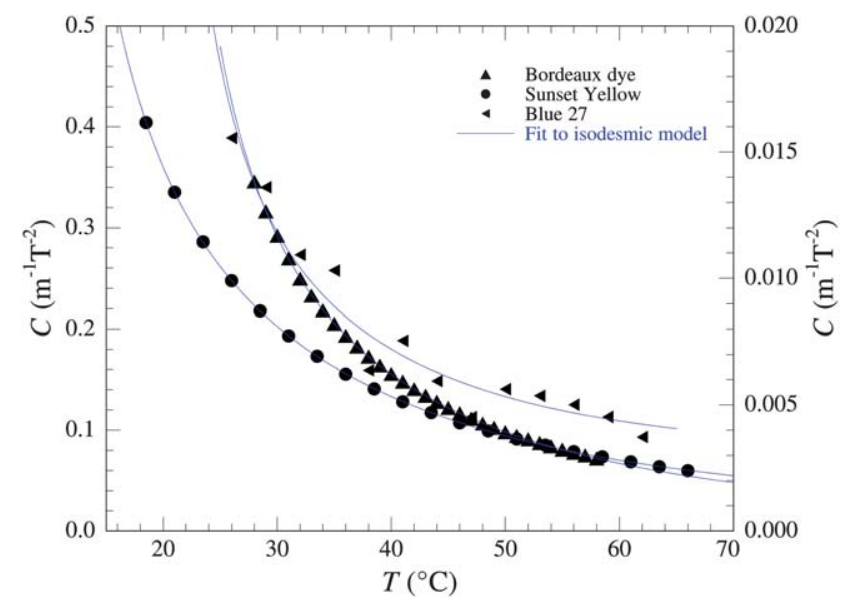

Fig. 7 (Color online) C versus $T$ for Blue 27, Bordeaux dye and Sunset Yellow, with fit to eqn (12). The data for Blue 27 are plotted against the scale on the right axis. It is apparent that the data for these three systems are described remarkably well by the isodesmic model. 
Bordeaux dye, and Blue 27. We also find that the fitted parameters $\alpha_{2}, \alpha_{3}$ are quite small, in the ranges -0.007 to -0.004 and 0.0003 to 0.0005 , respectively, for these LCLCs. This is consistent with only a minor evolution in $T^{*}$, due to gradual variation of mean aggregate length with temperature, and again indicates isodesmic behavior.

Next, we turn to our results for DSCG. As is evident from Fig. 8(a), the temperature dependence of $C(T)$ differs qualitatively from the other LCLC systems. While our measurements in the $12 \mathrm{wt} \%$ sample indicate a stronger decrease in $C(T)$ above

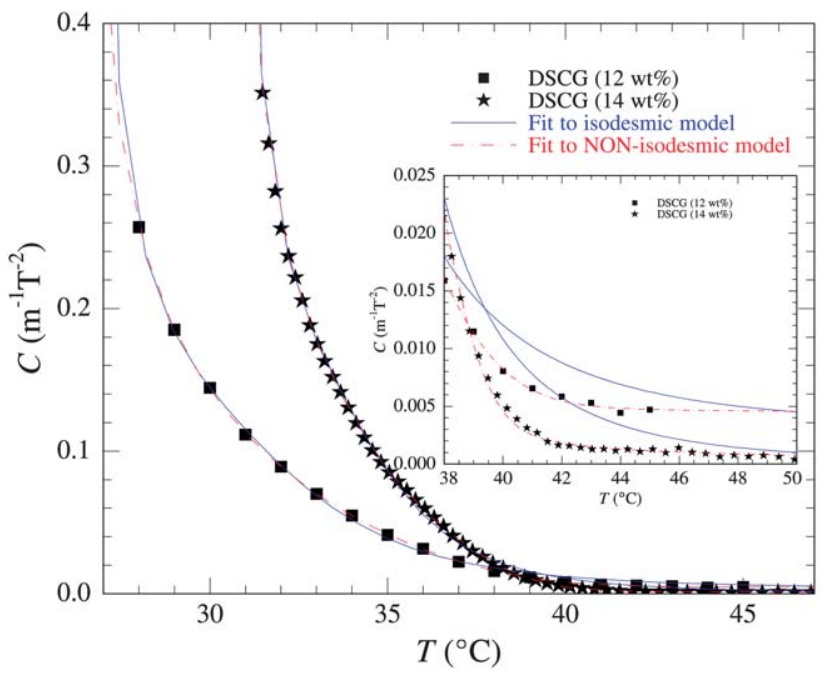

(a)

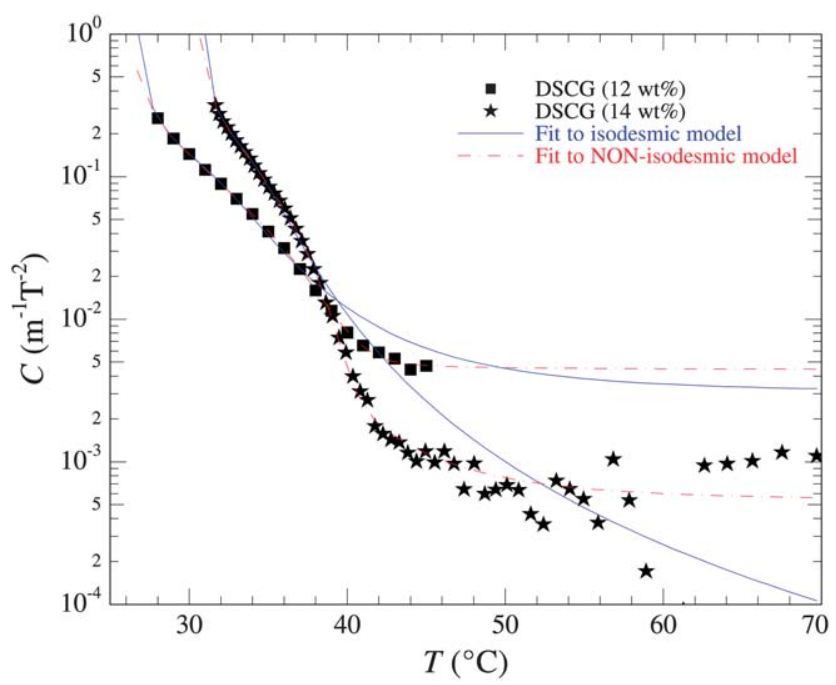

(b)

Fig. 8 (Color online) (a) C versus $T$ for $12 \mathrm{wt} \%$ and $14 \mathrm{wt} \%$ DSCG. The solid lines correspond to eqn (12) (isodesmic model), whereas the dotted lines correspond to eqn (12) using the more general model for $\bar{L} / d$ of eqn (13) (non-isodesmic model). While both models appear to describe the data prior to the sudden decrease to zero, the inset shows that the isodesmic model cannot fully describe the behavior of $C(T)$ far from $T_{N+1 .}$ (b) We can easily see the fit quality of the nonisodesmic model by examining the data on a $y$-axis logarithmic scale, where the data $T \gg T_{N+1}$ are only describable by including eqn (13).
$T_{N+I}$, more complete data, obtained on a $14 \mathrm{wt} \%$ sample, extending to higher temperatures, clearly establish the difference. These data, recorded during a temperature scan at fixed $B$ $=25$ Tesla, demonstrate that $\Delta n$ decreases rather rapidly with $T$ above $T_{N+I}$ to a minimum value that is consistent with zero within our experimental resolution, and then remains essentially flat with further increase in $T$. As further shown in Fig. 8(b) (utilizing a log scale for clarity), the standard (isodesmic) model for $\bar{L} / d$ in eqn (11), used with eqn (12), cannot account for the sharp decrease and flattening out of the data for $C(T)$ in the isotropic phase. To describe the unusual results for DSCG, we therefore propose a model for a non-isodesmic process, characterized by a strongly temperature-dependent $\bar{L} / d$.

Let us start by briefly reviewing the classical isodesmic model, which was developed years ago and, for example, describes the growth of worm-like micelles. ${ }^{30}$ The free energy density of the system, used to calculate a length distribution, is constructed from two parts: the non-extensive part of the free energy for the individual aggregates, taken simply to be $\sum_{L} E_{\mathrm{a}} c(L)$ (where $c(L)$ is the concentration of aggregates with length $L$ ), and the entropy of mixing for the aggregates, $k_{\mathrm{B}} T \sum_{L} c(L) \ln \left[c(L) \nu_{0}\right]$ (where $v_{0}$ is a molecular volume). Thus, the effective free energy density of the system is: $f=\sum_{L}\left(E_{\mathrm{a}} c(L)+\right.$ $\left.k_{\mathrm{B}} T c(L) \ln \left[c(L) \nu_{0}\right]\right)$. Minimization of this density over $c(L)$, subject to the constraint of fixed volume fraction $\phi$ of chromonic molecules $\left(\sum_{L} L c(L) \nu_{0}=\frac{\nu_{0}}{L_{0}} \int_{0}^{\infty} \mathrm{d} L L c(L)=L_{0} \phi\right), \quad$ gives $\quad c(L)=$ $\exp \left(-E_{\mathrm{a}} / k_{\mathrm{B}} T\right) \exp (-L / \bar{L}) /\left(e \nu_{0}\right)$, where $\bar{L}=L_{0} e^{1 / 2} \phi^{1 / 2} \exp \left(E_{\mathrm{a}} / 2 k_{\mathrm{B}} T\right)$. This is eqn (11) above.

To extend the model for the case of a non-isodesmic process, we consider the possibility that the aggregates may statistically occupy two states, one characterized by non-extensive energy $U_{0}$ $=E_{\mathrm{a}}$ and the other, a temperature-dependent "excited" state of the aggregate, characterized by energy $U_{1}=E_{\mathrm{a}}+\gamma k_{\mathrm{B}}\left(T_{\mathrm{c}}-T\right)$, where $\gamma$ and the temperature $T_{\mathrm{c}}$ are two phenomenological parameters. In this case, the non-extensive energy of an aggregate is given by $\frac{-\partial}{\partial \beta} \ln \left[\exp \left(-\beta U_{0}\right)+\exp \left(-\beta U_{1}\right)\right]$, or $E_{\mathrm{a}}+\frac{\gamma k_{\mathrm{B}}\left(T_{\mathrm{c}}-T\right)}{\exp \left[\gamma\left(T_{\mathrm{c}}-T\right) / T\right]+1}$. This replaces $E_{\mathrm{a}}$ in the previous expression for the free energy density $f$ of the system. We note that this new expression reduces to $E_{\mathrm{a}}$ if $\gamma \rightarrow 0$. The constrained minimization of $f$ now gives a more strongly temperaturedependent mean length. However, to ensure a finite $\bar{L}$ at high temperature, it is necessary to include a lower cutoff on the integration over $L$ in the constraint condition, i.e. $\sum_{L} \rightarrow \frac{1}{L_{0}} \int_{L_{1}}^{\infty} \mathrm{d} L$, where $L_{1}$ is chosen to ensure the same high temperature limit as eqn (11), namely, $\bar{L} \rightarrow L_{0} e^{1 / 2} \phi^{1 / 2}$ as $T \rightarrow \infty$. The final result for $\bar{L}$ is:

$$
\begin{aligned}
\frac{\bar{L}(T)}{d}= & \frac{L_{0}}{d} e^{1 / 2} \phi^{1 / 2}\left[\exp \left(\frac{E_{\mathrm{a}}}{k_{\mathrm{B}} T}\right) \exp \left(\frac{\gamma\left(T_{\mathrm{c}}-T\right) / T}{\exp \left[\gamma\left(T_{\mathrm{c}}-T\right) / T\right]+1}\right)\right. \\
& \left.-\exp \left(\frac{-\gamma}{\exp (-\gamma)+1}\right)+1\right]^{1 / 2}
\end{aligned}
$$


This expression reduces to eqn (11) when $\gamma \rightarrow 0$, but for large $\gamma$, it exhibits a sharp change with temperature in the vicinity of $T_{\mathrm{c}}$.

In Fig. 8, we present fits of the DSCG data for $C(T)$ for 12 and $14 \mathrm{wt} \%$ DSCG samples to eqn (12) and using a more general model for $\bar{L} / d$ in eqn (13) with $\gamma=540$ and $T_{\mathrm{c}}=308 \mathrm{~K}$. We also included a small, fixed baseline for $C(T)$, which represents an instrumental offset in our experiment. The model matches the data quite well and it clearly is more accurate than the simple isodesmic model. This result indicates a significantly more dramatic $T$ dependence for the length of aggregates in the isotropic phase of DSCG compared to that reflected in the data for the other LCLCs. Moreover, the values of the fit parameters $\alpha_{2}=-0.21$ and $\alpha_{3}=0.035$ are much larger in magnitude for DSCG than the other systems; this is expected if the variation of $\bar{L} / d$ is more dramatic. It should be stressed that our main qualitative conclusion is in fact independent of the details of the model leading to eqn (13): an accurate description of our data for DSCG requires a substantially stronger temperature dependence of $\bar{L} / d$ than that expected in a purely isodesmic aggregation process.

Unusual aggregation behavior in aqueous DSCG has also been deduced from recent light scattering ${ }^{\mathbf{1 4}}$ and optical absorption experiments. ${ }^{6}$ These experiments indicated that the aggregates become essentially isotropic in shape at a temperature $\sim 10^{\circ} \mathrm{C}$ above the coexistence region, which is roughly consistent with the decrease in $C(T)$ to essentially background values in Fig. 8(a). Our results encompassing four different LCLCs nevertheless confirm that non-isodesmic aggregation in LCLCs is atypical.

The difference in the aggregation properties of DSCG compared to the other systems is presumably a manifestation of some structural difference in the aggregates or of a possibly subtle difference in their thermodynamic and/or electrostatic stabilization. X-ray experiments on DSCG indicate that the cross sectional area of the aggregates is roughly equivalent to twice the area of single molecules, ${ }^{31}$ so side-side dimerization of molecules within the stack is a possible distinguishing feature for a non-isodesmic process. In fact, X-ray measurements on Sunset Yellow, which assembles isodesmically, point to columnar aggregates of single molecules. ${ }^{5,32}$ However, similar measurements ${ }^{19}$ indicate that Bordeaux dye breaks the pattern. In this case, aggregates with a two-molecule cross section develop isodesmically. The distribution of charges on the aggregates also does not yield a satisfactory explanation for the singular behavior of DSCG. Both DSCG and Bordeaux dye have charged groups on the same side, whereas Blue 27 and Sunset Yellow have charged groups on opposite sides, ${ }^{32}$ so it is unlikely that the reason for the difference in aggregation process between DSCG and the other three systems is merely due to the location of these charged groups. Perhaps the difference is molecular, given the higher flexibility of the core of the DSCG molecule relative to the other molecules.

\subsection{High-field behavior of the induced birefringence}

We now return to the question of the origin of the substantial

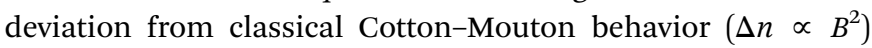

observed for certain LCLCs in Fig. 4 when $T$ is close to $T_{N+I}$ and at larger values of $B^{2}$. One possibility is that additional optical effects become significant in these cases, including Sunset Yellow and Bordeaux dye, but not in DSCG. Previous analyses, such as that presented by Luoma, ${ }^{13}$ assume that the media are weakly absorptive, in which case one may decompose the optical Jones matrix into the product of matrices for separate effects. In this analysis, linear dichroism cancels out in the measurement of the optical phase shift $\varphi$ and thus cannot lead to the nonlinearity of $\Delta n\left(B^{2}\right)$ seen in Fig. $4(\mathrm{~b})$ and (d). The general form of the Jones matrix ${ }^{33,34}$ for an optically anisotropic material contains eight independent parameters describing eight possible optical properties. Two of them correspond to the isotropic mean refraction and mean absorption. The other six parameters describe linear and circular birefringence, linear and circular dichroism, and the so-called Jones birefringence and Jones dichroism.

Separate, direct measurements verified that both linear and circular dichroism (in the materials we studied) are below our threshold of detection. Two other effects, the so-called Jones birefringence and dichroism (linear birefringence and dichroism, both oriented at $45^{\circ}$ to the optic axis), are extremely esoteric; it is not entirely clear that they are allowed,,$^{34}$ nor can they be measured independently. Furthermore, it is highly unlikely that the Jones dichroism should be taken into account when the linear and circular dichroism are ruled out.

In uniaxial nematics, the Jones dichroism is expected to be nonzero when the linear dichroism is nonzero. For a uniaxial nematic, the Jones birefringence does not refer to a separate material property, but appears when the optic axis of a crystalline sample is azimuthally misaligned with respect to the coordinate system, such that $\varphi_{\mathrm{JB}}=\varphi_{\mathrm{LB}} \tan 2 \alpha$, where $\alpha$ is the angle of misalignment, and $\varphi_{\mathrm{JB}}$ and $\varphi_{\mathrm{LB}}$ are the phase retardations due to the Jones birefringence and linear birefringence, respectively. ${ }^{33}$ For more details, see ref. 35 and 36. In our experiment, due to the negative diamagnetic anisotropy of the aggregates, they tend to align their long axes perpendicular to $\vec{B}$ without any preference for their direction within a plane $P \perp \vec{B}$. In other words, the polar angle between the optic axis and $\vec{B}$ is fixed, but the azimuthal orientation of the optic axis within the plane $P \perp \vec{B}$ is degenerate.

This is a rare example of the realization of a nematic-like structure with the scalar orientational order parameter $-\frac{1}{2}<S<0$. For $S=-\frac{1}{2}$, the optic axis induced by the applied magnetic field is strictly localized within the plane $P$ and thereby $\alpha=0$. For other values of $S$ from the interval $-\frac{1}{2}<S<0$, $\alpha$ is nonzero, but decreases for the increasing magnetic field, which implies that $\varphi_{\mathrm{JB}}$ also decreases when $B$ increases. If the observed deviation from the Cotton-Mouton law were due to the Jones birefringence, then this deviation would decrease for increasing magnetic field, which is in conflict with our observation. Therefore, we opted to neglect four of the six anisotropic optical effects, namely: linear, circular and Jones dichroisms, and Jones birefringence, leading us to examine whether the two remaining effects, linear and circular birefringence, can adequately describe our data. 
Finally, we also made careful efforts to rule out instrumental effects - specifically, the possibility that the modulation amplitude setting could differ from the actual amplitude, which can affect the shape of $\varphi$ in the vicinity of $\varphi=n \pi / 2$, where $n$ is an integer. We developed a procedure to self-consistently correct for differences between the set and actual amplitudes, the details of which are described in ref. 21. To validate this procedure, we measured the magnetic field-induced optical phase difference in the isotropic state of a standard calamitic liquid crystal $(5 \mathrm{CB})$ from 0 to almost $1.6 \pi$ radians. The results, displayed in Fig. 9, show (within experimental uncertainty) ${ }^{37}$ that $\varphi$ increases linearly with $B^{2}$ as expected, which confirms that the unusual behavior we observe in these LCLCs is not an instrumental artifact.

Then, considering only a combination of linear and circular birefringence, the Jones matrix representing our LCLC samples becomes:

$$
\left(\begin{array}{cc}
\cos \frac{\Psi}{2}-\frac{i \varphi_{\mathrm{LB}}}{\Psi} \sin \frac{\Psi}{2} & \frac{\varphi_{\mathrm{CB}}}{\Psi} \sin \frac{\Psi}{2} \\
-\frac{\varphi_{\mathrm{CB}}}{\Psi} \sin \frac{\Psi}{2} & \cos \frac{\Psi}{2}+\frac{i \varphi_{\mathrm{LB}}}{\Psi} \sin \frac{\Psi}{2}
\end{array}\right)
$$

where $\varphi_{\mathrm{LB}}$ and $\varphi_{\mathrm{CB}}$ are the phase differences between orthogonal linear and circular components of transmitted light, respectively, and $\Psi=\sqrt{\varphi_{\mathrm{LB}}{ }^{2}+\varphi_{\mathrm{CB}}{ }^{2}}$. In the usual way, we may obtain the transmitted electric field vector $\vec{E}$ by multiplying together the matrices representing each optical element in our experimental setup, including the incident polarizer, sample, photoelastic modulator, and analyzer. The transmitted intensity is then evaluated by taking $\vec{E} \cdot \vec{E}^{*}$. Expanding the intensity in terms of Fourier modes of modulator frequency, we find for the ratio of lock-in amplifier signal voltages proportional to the first- and second harmonic components of the intensity:

$$
\frac{V_{1 f}}{V_{2 f}}=\frac{J_{1}(A)}{J_{2}(A)} \frac{\varphi_{\mathrm{LB}}}{\sqrt{\varphi_{\mathrm{LB}}^{2}+\varphi_{\mathrm{CB}}^{2}}} \tan \left(\sqrt{\varphi_{\mathrm{LB}}{ }^{2}+\varphi_{\mathrm{CB}}^{2}}\right) .
$$

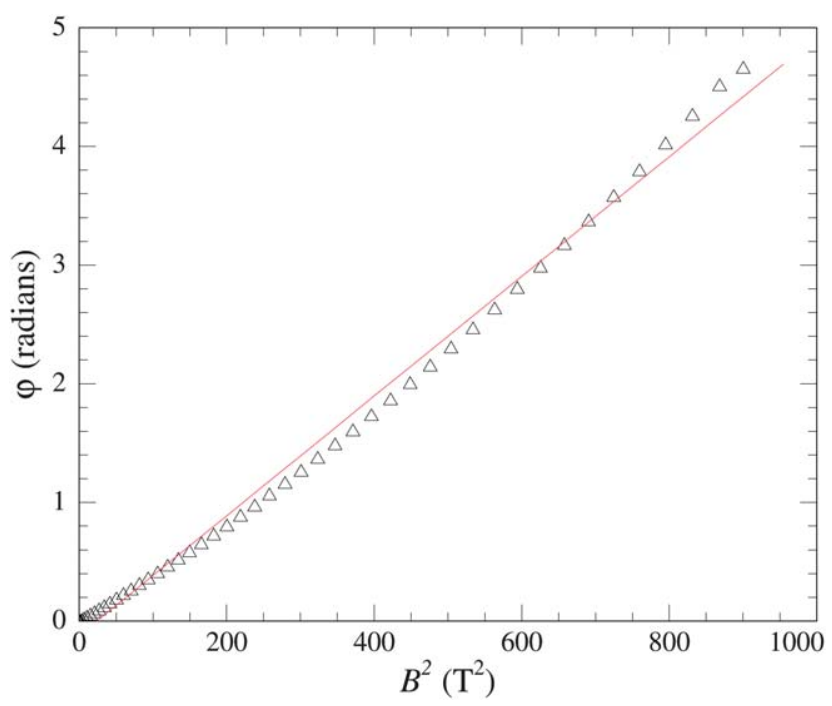

Fig. 9 (Color online) $\varphi$ versus $B^{2}$ for $5 C B$, with a linear fit super-imposed. The residual non-linearity is attributable to the resolution of the PEM. ${ }^{37}$ where $V_{1 f}$ and $V_{2 f}$ are the first- and second harmonic components of the optical signal, $f$ is the photoelastic modulation frequency, and $J_{1}(\mathrm{~A})$ and $J_{2}(\mathrm{~A})$ are the Bessel function of the first and second kinds, respectively, evaluated at $A$, the amplitude of optical phase modulation.

Recalling that $\varphi=2 \pi d \Delta n / \lambda$, we make the following substitutions into eqn (15): $\varphi_{\mathrm{LB}}=2 \pi d \Delta n_{\mathrm{LB}} / \lambda$ and $\varphi_{\mathrm{CB}}=2 \pi d \Delta n_{\mathrm{CB}} / \lambda$, where $\Delta n_{\mathrm{LB}}=n_{\mathrm{e}}-n_{\mathrm{o}}$ is the refractive index difference between the extraordinary and ordinary linearly-polarized waves, and $\Delta n_{\mathrm{CB}}=n_{\mathrm{L}}-n_{\mathrm{R}}$ is the refractive index difference between the left circularly-polarized and right circularly-polarized waves. Note that, when $\varphi_{\mathrm{CB}}=0$, we obtain the usual result for when there is only field-induced linear birefringence. We also note that fieldinduced linear birefringence is simply the Cotton-Mouton effect described by eqn (1), and so $C \approx C_{\mathrm{LB}}$.

Finally, we use eqn (1) to insert the expected $B^{2}$ dependence, and arrive at the expression for the total phase shift:

$$
\tan \varphi=\frac{C_{\mathrm{LB}}}{\sqrt{C_{\mathrm{LB}}^{2}+C_{\mathrm{CB}}^{2}}} \tan \left(2 \pi B^{2} d \sqrt{C_{\mathrm{LB}}{ }^{2}+C_{\mathrm{CB}}^{2}}\right)
$$

where $C_{\mathrm{LB}}$ and $C_{\mathrm{CB}}$ are the $\mathrm{CM}$ coefficients due to induced $\Delta n_{\mathrm{LB}}$ and $\Delta n_{\mathrm{CB}}$, respectively, and $d$ is the path length through the sample. In eqn (16), the $T$ dependence of $C_{\mathrm{LB}}$ and $C_{\mathrm{CB}}$ is not written explicitly. We used this functional form to fit the data in Fig. 4(b) and (d), using only two adjustable parameters: $C_{\mathrm{LB}}$ and $C_{\mathrm{CB}}$. The fits are presented in Fig. 10 and the temperature dependence of the fit parameters is shown in Fig. 11. Since it was not possible to induce a sufficiently large phase difference in Blue 27, due to absorption, we could not detect any effect of $C_{\mathrm{CB}}$. Additionally, since the $1 \mathrm{~mm}$ path length DSCG sample also did not have a large phase difference, we also tested a $10 \mathrm{~mm}$ thick sample of DSCG. The two coefficients $C_{\mathrm{LB}}$ and $C_{\mathrm{CB}}$ for DSCG were determined using the thicker sample and appear in Fig. 11. Measurements on a sufficiently thick sample of Blue 27 could not be made due to the high absorption of this dye at the optical wavelength used. Note that for small $B^{2}$ and $\varphi$, eqn (16) reduces to $\varphi=2 \pi d C_{\mathrm{LB}} B^{2}$, or from the relation $\varphi=2 \pi d \Delta n / \lambda, \Delta n=C_{\mathrm{LB}} \lambda B^{2}$, which is identical to eqn (1). Thus, the linear in $B^{2}$ analysis of the measured $\Delta n$ at low $B^{2}$ should yield $C_{\mathrm{LB}}$, the quantity discussed in Section 3.1 above.

This analysis, including our correction procedure for instrumental effects, validated in Fig. 9 for phase shifts exceeding the largest measured in Fig. 10, indicates that nonzero values of both $\varphi_{\mathrm{LB}}$ and $\varphi_{\mathrm{CB}}$ are induced by the applied field. Since there is no broken chiral symmetry on a molecular level, and the geometry of the magnetic field is not correct to induce net non-zero circular birefringence (i.e. our magnetic field is perpendicular rather than parallel to the light propagation direction), this result is surprising. Interestingly, since in this case $\varphi_{\mathrm{LB}} \neq 0$, the relevant quantity in eqn (14) is $\varphi_{\mathrm{CB}}{ }^{2}$ rather than $\varphi_{\mathrm{CB}}$. Even though by symmetry $\varphi_{\mathrm{CB}}$, integrated over the optical path length, must be zero, we have an intriguing possibility; this zero average can be the result of a distribution of domains having both negative and positive CB.

However, what might be the origin of such regions? One obvious candidate is chirally stacked aggregates having a twisted distribution of their optic axis along the light path, 


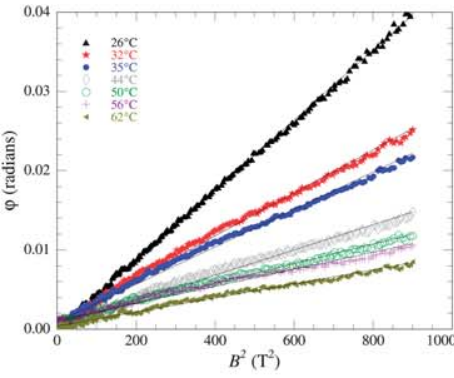

(a)

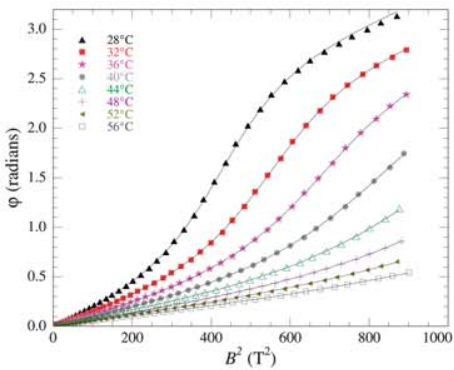

(b)

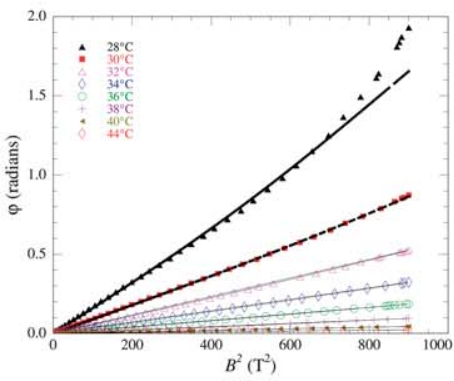

(c)

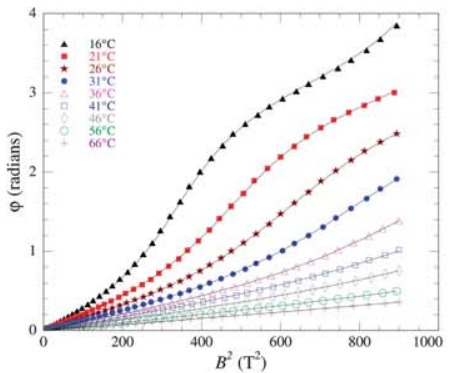

(d)

Fig. 10 (Color online) Fits for $\varphi$ versus $B^{2}$. (a) Blue 27, (b) Bordeaux dye, (c) DSCG, and (d) Sunset Yellow, assuming that both linear birefringence and circular birefringence are induced by the field.

although with both handedness occurring with equal probability. Such aggregates, or perhaps groups of such aggregates, would necessarily be of size at least comparable to $\lambda$ in order to produce the effects discussed above. Chirality in the aggregates could be induced due to a shift in the charge density on each molecule in the aggregate, thus forcing a helical offset of the molecular planes as they stack. Then, $\varphi_{\mathrm{CB}}$ is the difference between the azimuths of the long axes of the light polarization

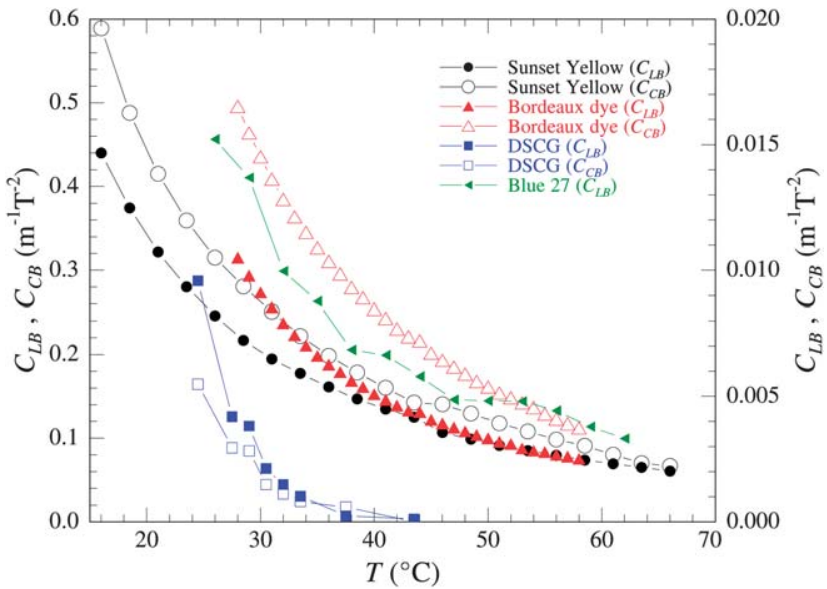

Fig. 11 (Color online) $C_{\mathrm{LB}}$ and $C_{\mathrm{CB}}$ (where applicable) versus $T$ for all four systems. The data for Blue 27 are plotted against the scale on the right axis and the lines shown are used to connect the points.

ellipses at the exit and entrance interfaces of the sample. The contributions to the rotation of the light polarization plane due to a random spatial distribution of the left- and right-handed species along the light path average to zero, leading to $\varphi_{\mathrm{CB}}$ averaging to zero. Indeed, small scale chirality has been confirmed in tactoidal nuclei of the nematic phase of DSCG coexisting with its isotropic melt. ${ }^{38}$

The observed nonzero $\varphi_{\mathrm{CB}}{ }^{2}$ suggests that the system demonstrates macroscopic chirality over spatial scales comparable at least to $\lambda$. Such a macroscopic effect may arise from chromonic aggregates interacting with each other. One of the mechanisms of long-range interactions is through the counterions surrounding the chromonic aggregates. We recall that the chromonic aggregates are essentially polyions, with the surface groups becoming ionized in water. The positive surface charges are screened by the clouds of negatively charged counterions. The presence of surface charges and equal number of counterions (the overall charge is zero) greatly modifies the interactions of particles in colloidal systems, as was recognized already by Onsager in his model of the excluded volume-induced nematic phase. ${ }^{39}$

In particular, a rod-like, non-chiral polyion exerts long-range orientational torques on its similarly charged rod-like polyions. Interestingly, the sign of this effect depends on how the interactions are treated. In the mean-field theory, the interactions are predicted to lead to a perpendicular arrangement of charged rods; ${ }^{40}$ in the Debye-Hückel plasma approach, the theory predicts parallel orientation of the rods. ${ }^{41}$ It is reasonable to assume that the counterion clouds around the chiral aggregates would facilitate long-range correlations, "transferring" chirality from individual aggregates to their assemblies. The existence of chiral torques has been demonstrated in counterion-mediated electrostatic interactions between helical DNA molecules separated by distances of a few $\lambda_{\mathrm{D}} ;^{\mathbf{4 2}}$ electrostatically, the LCLC aggregates are similar to DNA, carrying 6 ionizable sites per 1 $\mathrm{nm}$ of the aggregate length.

The second mechanism of macroscopic chirality in chromonic systems, already confirmed experimentally for the fieldfree biphasic region in which the nematic and isotropic phases 
coexist ${ }^{38}$ is rooted in the strong elastic anisotropy of the nematic ordering of LCLCs. As measured for Sunset Yellow, the elastic constant $K_{22}$ of twist is about one order of magnitude smaller than the elastic constants of splay $K_{11}$ and bend $K_{33} \cdot{ }^{43}$ This elastic anisotropy controls the director field inside the nuclei (tactoids) of the nematic phase appearing in the isotropic fluid. The director is necessarily distorted because of the closed shape of the tactoids and because of the surface anchoring that requires the director to be tangential to the nematic-isotropic interface. ${ }^{38}$ The smallness of $K_{22}$ leads to macroscopic twist deformations that relieve splay and bend distortions. ${ }^{38}$ Within each tactoid, the sense of the twist is either left- or right-handed, but it alternates from one tactoid to the next.

In these systems, the typical screening length (Debye length $\lambda_{\mathrm{D}}$ ) ranges from about $0.3 \mathrm{~nm}$ for Sunset Yellow ${ }^{44}$ to $0.5 \mathrm{~nm}$ for DSCG. ${ }^{45}$ These values of $\lambda_{\mathrm{D}}$ are comparable to the diameter of the aggregates (1.2 $\mathrm{nm}$ for Sunset Yellow) and to the distances between the surfaces of neighboring aggregates. Assuming that each Sunset Yellow aggregate has a radius of $0.6 \mathrm{~nm}$ and a length of $1 \mathrm{~nm}$, with a volume fraction of about 0.2 , the centerto-center distance between the aggregates is about $1.8 \mathrm{~nm}$; therefore, the surface-to-surface distance is only about $0.6 \mathrm{~nm}$, comparable to the above estimates of $\lambda_{\mathrm{D}}$. The orientational torques between the charged chromonic aggregates might lead to macroscopic amplification of a single-aggregate chirality. To verify this hypothesis, it would be extremely interesting to explore the behavior of LCLCs doped with different amount of salts; large concentrations of salts might reduce $\lambda_{\mathrm{D}}$ and mitigate the effect of the field induced $\varphi_{\mathrm{CB}}$.

As was previously noted, the induced optic axis tends to align parallel to the plane $P \perp \vec{B}$. There is no long range correlation in the orientations of the aggregates when moving along any direction perpendicular to $\vec{B}$, including that along the light path. The azimuthal orientation of the optic axes within the plane $P$ is assumed to be random at long distances, with respect to the inter-aggregate distances, but there are short range orientation fluctuations forming clusters with nematic-like ordering inside them and without any correlation between them. The light will be scattered by these clusters and partially depolarized. Partial depolarization of the light implies that the azimuth of the light wave at the exit sample interface will be randomly rotated across the light beam cross-section with respect to the entrance polarization direction. Therefore, the value and sign of the light polarization rotation will be a function $\varphi_{\mathrm{CB}}(x, z)$ of the coordinates $(x, z)$ across the light beam (see Fig. 2 for the definition of the coordinate system). Nonzero $\varphi_{\mathrm{CB}}(x, z)$ contributes as nonzero circular birefringence to the total phase retardation in eqn (15).

Since the applied magnetic field only fixes the polar angle between the optic axis and $\vec{B}$, but has no impact on the azimuthal angle of the optic axis orientation along the light beam because the light propagation direction is perpendicular to $\vec{B}$, the light scattering, and thus the light depolarization along the light path, should not depend on $B$, but should increase on cooling. Therefore, the light depolarization due to scattering might be the origin of nonzero circular birefringence $\varphi_{\mathrm{CB}}$ in eqn (15) and therefore nonzero $C_{\mathrm{CB}}$ in eqn (16), explaining the observed deviation from the Cotton-Mouton law. The fact that the deviation from the Cotton-Mouton law is well pronounced for Sunset Yellow and Bordeaux dye and is much less for DSCG can be due to the stronger light depolarization in the light absorbing materials.

\section{Conclusions}

The nature of field induced birefringence at temperatures above the (zero-field) nematic-isotropic two-phase coexistence region yields valuable insight into the nature of aggregation in LCLC suspensions. Specifically, the temperature dependence of the Cotton-Mouton coefficient is not only substantially more complicated than is predicted by simple Landau-deGennes theory, it also differs markedly from one material to another. We find a distinct difference between the aggregation behavior of disodium cromoglycate solutions compared to the other three materials studied: DSCG cannot be adequately described by models assuming an isodesmic aggregation process. In addition, we have extended our measurements to very high magnetic fields, and corresponding optical phase differences. In these regimes, we observed some remarkable and unexpected behavior, specifically a non-zero result for induced circular birefringence in a non-chiral material. We have discussed possible origins of this effect, but further work is required in order to definitively establish how this result arises.

\section{Acknowledgements}

The authors acknowledge invaluable assistance from S. McGill, M. Omelchenko, and W. Aldhizer. This work was supported by the NSF (DMR-0606160, DMR-0964765, and DMR-1121288), the Ohio Board of Regents, and grant 0113U000468 from the Ministry of Education and Science of Ukraine. Work performed at NHMFL supported by NSF cooperative agreement DMR0084173, the State of Florida and the U.S. Department of Energy.

\section{References}

1 J. Lydon, Curr. Opin. Colloid Interface Sci., 2004, 8, 480-490.

2 S.-W. Tam-Chang and L. Huang, Chem. Commun., 2008, 1957-1967.

3 Handbook of Liquid Crystals, ed. D. Demus, J. Goodby, G. Gray, H.-W. Spiess and V. Vill, Wiley-VCH, 1998, vol. 2B.

4 J. Howell, J. Allergy Clin. Immunol., 2005, 115, 882.

5 V. Horowitz, L. Janowitz, A. Modic, P. Heiney and P. Collings, Phys. Rev. E: Stat., Nonlinear, Soft Matter Phys., 2005, 72, 041710.

6 P. Collings, A. Dickinson and E. Smith, Liq. Cryst., 2010, 37, 701-710.

7 J. Lydon, Liq. Cryst., 2011, 38, 1663-1681.

8 H.-S. Park and O. Lavrentovich, Lyotropic Liquid Crystals: Emerging Applications, in Liquid Crystals Beyond Displays, Wiley-VCH, 2012, ch. 14.

9 P. Maiti, Y. Lansac, M. Glaser and N. Clark, Liq. Cryst., 2002, 29, 619-626. 
10 Y. A. Nastishin, H. Liu, S. Shiyanovskii, O. Lavrentovich, A. Kostko and M. Anisimov, Phys. Rev. E: Stat., Nonlinear, Soft Matter Phys., 2004, 70, 051706.

11 R. Guenther, Modern Optics, John Wiley and Sons, 1990.

12 J. Champion and G. Meeten, J. Pharm. Sci., 1973, 62, 1589.

13 R. Luoma, Ph.D. thesis, Brandeis University, 1995.

14 C. Bertrand, K. Linegar, A. Kostko and M. Anisimov, Phys. Rev. E: Stat., Nonlinear, Soft Matter Phys., 2009, 79, 041704.

15 P. de Gennes and J. Prost, The Physics of Liquid Crystals, Oxford University Press, 1993.

16 R. Vasyuta, O. Boiko, Y. Piryatinskiy, V. Nazarenko and A. Kachkovsky, Mol. Cryst. Liq. Cryst., 2005, 426, 117-127.

17 D. Edwards, J. Jones, O. Lozman, A. Ormerod, M. Sintyureva and G. Tiddy, J. Phys. Chem., 2008, 112, 14628-14636.

18 Y. A. Nastishin, H. Liu, T. Schneider, V. Nazarenko, R. Vasyuta, S. Shiyanovskii and O. Lavrentovich, Phys. Rev. E: Stat., Nonlinear, Soft Matter Phys., 2005, 72, 041711.

19 M. Tomasik and P. Collings, J. Phys. Chem. B, 2008, 112, 9883-9889.

20 J. Kemp, J. Opt. Soc. Am., 1969, 59, 950-954.

21 T. Ostapenko, Ph.D. thesis, Kent State University, 2011.

22 D. Wiant, Ph.D. thesis, Kent State University, 2007.

23 J. Tang and S. Fraden, Phys. Rev. Lett., 1993, 71, 3509-3512.

24 B. Lemaire, P. Davidson, J. Ferré, J. Jamet, P. Panine, I. Dozov and J. Jolivet, Phys. Rev. Lett., 2002, 88, 125507.

25 D. van der Beek, A. Petukhov, P. Davidson, J. Ferré, J. Jamet, H. Wensink, G. Vroege, W. Bras and H. Lekkerkerker, Phys. Rev. E: Stat., Nonlinear, Soft Matter Phys., 2006, 73, 041402.

26 W. Bragg and A. Pippard, Acta. Cryst., 1953, 6, 865-867.

27 P. Barois and F. Nallete, J. Phys. II, 1994, 4, 1049.

28 M. Born and E. Wolf, Principles of Optics, Cambridge University Press, 7th edn, 1999.

29 J. Osborn, Phys. Rev., 1945, 67, 351.

30 M. Cates and S. Candau, J. Phys.: Condens. Matter, 1990, 2, 6869.
31 N. Hartshorne and G. Woodard, Mol. Cryst. Liq. Cryst., 1973, 23, 343-368.

32 A. Dickinson, N. LaRacuente, C. McKitterick and P. Collings, Mol. Cryst. Liq. Cryst., 2009, 509, 9-20.

33 R. Jones, J. Opt. Soc. Am., 1948, 38, 671-683.

34 O. Arteaga and A. Canillas, Opt. Lett., 2010, 35, 559-561.

35 Y. A. Nastishin and S. Y. Nastyshyn, Ukr. J. Phys. Opt., 2011, 12, 191-201.

36 Y. A. Nastishin and S. Y. Nastyshyn, Phys. Rev. A: At., Mol., Opt. Phys., 2013, 87, 033810.

37 In order to be perfectly linear, the optical phase amplitude modulation must be set precisely to a zero of the zeroth order Bessel function of the first kind (i.e. 2.40482). The PEM precision on setting the phase amplitude is only 0.006; this imprecision is sufficient to cause the deviation from linearity observed in $5 \mathrm{CB}$.

38 L. Tortora and O. Lavrentovich, Proc. Natl. Acad. Sci. U. S. A., 2011, 108, 5163-5168.

39 L. Onsager, Ann. N. Y. Acad. Sci., 1949, 51, 627.

40 A. Stroobants, H. Lekkerkerker and T. Odijk, Macromolecules, 1986, 19, 2232-2238.

41 I. Potemkin, N. Oskolkov, A. Khokhlov and P. Reineker, Phys. Rev. E: Stat., Nonlinear, Soft Matter Phys., 2005, 72, 021804.

42 A. Kornyshev, D. Lee, S. Leikin and A. Wynveen, Rev. Mod. Phys., 2007, 79, 943-996.

43 S. Zhou, Y. A. Nastishin, M. Omelchenko, L. Tortora, V. Nazarenko, O. Boiko, T. Ostapenko, T. Hu, C. Almasan, S. Sprunt, J. Gleeson and O. Lvrentovich, Phys. Rev. Lett., 2012, 109, 037801.

44 H.-S. Park, S.-W. Kang, L. Tortora, S. Kumar and O. Lavrentovich, Langmuir, 2011, 27, 4164-4175.

45 L. Tortora, H.-S. Park, S.-W. Kang, V. Savaryn, S.-H. Hong,

K. Kaznatcheev, D. Finotello, S. Sprunt, S. Kumar and O. Lavrentovich, Soft Matter, 2010, 6, 4157-4167. 